\title{
Cosmological magnetic fields from inflation in extended electromagnetism
}

\author{
Jose Beltrán Jiménez and Antonio L. Maroto \\ Departamento de Física Teórica, Universidad Complutense de Madrid, 28040, Madrid, Spain
}

(Received 26 May 2010; published 19 January 2011)

\begin{abstract}
In this work we consider an extended electromagnetic theory in which the scalar state which is usually eliminated by means of the Lorenz condition is allowed to propagate. This state has been shown to generate a small cosmological constant in the context of standard inflationary cosmology. Here we show that the usual Lorenz gauge-breaking term now plays the role of an effective electromagnetic current. Such a current is generated during inflation from quantum fluctuations and gives rise to a stochastic effective charge density distribution. Because of the high electric conductivity of the cosmic plasma after inflation, the electric charge density generates currents which give rise to both vorticity and magnetic fields on sub-Hubble scales. Present upper limits on vorticity coming from temperature anisotropies of the $\mathrm{CMB}$ are translated into lower limits on the present value of cosmic magnetic fields. We find that, for a nearly scale invariant vorticity spectrum, magnetic fields $B_{\lambda}>10^{-12} \mathrm{G}$ are typically generated with coherence lengths ranging from subgalactic scales up to the present Hubble radius. Those fields could act as seeds for a galactic dynamo or even account for observations just by collapse and differential rotation of the protogalactic cloud.
\end{abstract}

DOI: 10.1103/PhysRevD.83.023514

PACS numbers: 98.80.-k, 12.20. $-\mathrm{m}$, 98.62.En

Traditionally it has been argued that due to the electric neutrality of the universe on large scales, the only relevant interaction in cosmology should be gravitation. However, the behavior of electromagnetic fields on astrophysical and cosmological scales is still far from clear, the most evident example being the unknown origin of magnetic fields observed in galaxies and galaxy clusters.

Magnetic fields with large coherence lengths (around $10 \mathrm{kpc}$ or even larger) and strengths around $10^{-6} \mathrm{G}$ [1] have been measured in galaxies of all types and in galaxy clusters located at very different redshifts. Also, recent works [2] show evidence for the existence of strong extragalactic magnetic fields above $3 \times 10^{-16} \mathrm{G}$ with coherence lengths much larger than the cluster scales and eventually reaching the present Hubble radius.

Two different types of scenarios have been considered for the generation of such fields. On one hand is the primordial field hypothesis, i.e. the existence of relic magnetic fields from the early Universe with comoving strengths around $10^{-10}-10^{-12} \mathrm{G}$ which permeated the protogalactic medium and were amplified to the present values by collapse and differential rotation. On the other hand, we have the dynamo mechanism, in which much weaker fields, around $10^{-19} \mathrm{G}$ [3], could have been amplified by the galactic rotation. However, it is known that the latter scenario has certain limitations since the time scales for dynamo amplification may be too long to explain the observed fields in young objects [4].

Both scenarios require preexisting seed fields to be amplified and proposals for their generation include astrophysical mechanisms [5], production during inflation [6], in phase transitions [7], and others [8]. Although some of those mechanisms could seed a galactic dynamo, the generation of the stronger seeds required in the primordial field hypothesis is much more problematic. In any case, according to [2], astrophysical processes, generation in phase transitions or during recombination, could not explain the claimed extragalactic detection, and, in principle, only production during inflation could account for observations. Generation of magnetic fields during inflation requires the breaking of the conformal triviality of standard electromagnetism in a Robertson-Walker background. For that reason, modified electromagnetic theories, including nonminimal curvature couplings or couplings to extra fields such as the inflaton or dilaton, have been studied in the literature [6].

Recently, the possibility of producing a small cosmological constant during inflation in the context of an extended electromagnetic model has been considered in [9-11]. The proposed theory involves a modification of the nontransverse electromagnetic sector, which breaks conformal triviality but respects the ordinary (transverse) photon dynamics. Unlike previous models, the modified equations remain linear, without potential terms, dimensional parameters, or explicit curvature couplings. The aim of this work will be to explore the possibility that largescale cosmic magnetic fields could be generated in this extended theory.

Let us start by writing the generalized electromagnetic action which includes, apart from the coupling to the conserved current $J^{\mu}$, a gauge-breaking term $[9,10]$ :

$$
S=\int d^{4} x \sqrt{g}\left[-\frac{1}{4} F_{\mu \nu} F^{\mu \nu}+\frac{\xi}{2}\left(\nabla_{\mu} A^{\mu}\right)^{2}+A_{\mu} J^{\mu}\right] .
$$

Because of the presence of the gauge-breaking term, this action does not respect the invariance under arbitrary 
gauge transformation, but it still preserves a residual gauge symmetry given by $A_{\mu} \rightarrow A_{\mu}+\partial_{\mu} \theta$ provided $\square \theta=0$.

The corresponding modified Maxwell equations read

$$
\nabla_{\nu} F^{\mu \nu}+\xi \nabla^{\mu}\left(\nabla_{\nu} A^{\nu}\right)=J^{\mu} .
$$

Taking the 4-divergence of these equations we obtain

$$
\square\left(\nabla_{\nu} A^{\nu}\right)=0,
$$

where we have used the fact that the electromagnetic current is covariantly conserved.

Thus we see that due to the presence of the $\xi$ term, the free theory contains three propagating physical fields, which correspond to the two ordinary transverse photons and a third scalar state related to $\nabla_{\nu} A^{\nu}$ (in principle, we should include a fourth polarization for $A_{\mu}$; however, it can be seen to correspond to the pure gauge mode $\partial_{\mu} \theta$ ). Notice that in the ordinary approach to electrodynamics [12] the same action (1) is considered, but $\nabla_{\nu} A^{\nu}$ is imposed to be zero (Lorenz condition), so that we are left only with the two transverse polarizations. However, in the modified approach we will follow, we allow this state to propagate. Despite the fact that the extended theory is not gauge invariant, the transverse photon dynamics is not affected and remains gauge invariant. This implies that ordinary QED phenomenology is recovered in Minkowski spacetime. On the other hand, the fact that the theory contains an additional polarization could suggest the possibility that such a mode is a ghost and the theory would be quantummechanically unstable. However, as shown in [10], thanks to the residual gauge symmetry of the theory it is possible to eliminate the ghost state, so that the new mode has a positive norm (details on theoretical and experimental aspects of the theory can be found in [9-11], and references therein).

As seen from (3) the new state is completely decoupled from the conserved currents, although it is nonconformally coupled to gravity. This means that this state cannot be excited from electromagnetic currents. However, it could be produced from quantum fluctuations in a curved spacetime, in a similar way as inflaton fluctuations during inflation. Moreover, due to the well-known fact that a massless scalar field gets frozen on super-Hubble scales for a Robertson-Walker universe $\left[d s^{2}=a^{2}(\eta)\left(d \eta^{2}-d \vec{x}^{2}\right)\right]$, we get $\nabla_{\nu} A^{\nu} \sim$ const on scales larger than the Hubble radius, giving rise to a cosmological-constant-like term in the action (1). This constant has been shown to agree with observations provided inflation took place at the electroweak scale $[9,10]$. On the other hand, for sub-Hubble scales, we have that $\nabla_{\nu} A^{\nu} \sim a^{-1} e^{i(k \eta-\vec{k} \vec{x})}$.

It is interesting to note that the $\xi$ term can be seen, at the equations of motion level, as a conserved current acting as a source of the usual Maxwell field. To see this, we can write $-\xi \nabla^{\mu}\left(\nabla_{\nu} A^{\nu}\right) \equiv J_{\nabla \cdot A}^{\mu}$, which, according to (3), satisfies the conservation equation $\nabla_{\mu} J_{\nabla \cdot A}^{\mu}=0$, and we can express (2) as

$$
\nabla_{\nu} F^{\mu \nu}=J_{T}^{\mu}
$$

with $J_{T}^{\mu}=J^{\mu}+J_{\nabla \cdot A}^{\mu}$ and $\nabla_{\mu} J_{T}^{\mu}=0$. Physically, this means that, while the new scalar mode can only be excited gravitationally, once it is produced it will generally behave as a source of electromagnetic fields. Therefore, the modified theory is described by ordinary Maxwell equations with an additional "external" current.

In the following we will study the phenomenological consequences of the presence of this new effective current. We will show that it can be generated during inflation, and we will compute its corresponding power spectrum. This implies that the universe will acquire a nonvanishing Gaussian stochastic distribution of effective electric charge with zero mean but a nonvanishing dispersion. This, in vacuum, could also be seen as the generation of a stochastic background of longitudinal electric waves. Because of the high electric conductivity after inflation, an electrically charged universe has been shown to lead necessarily to the generation of vorticity and the presence of magnetic fields on cosmological scales $[13,14]$. Notice that, even though conductivity will be in general high after reheating also in ordinary electromagnetism, the presence of a nonvanishing effective charge density is the crucial ingredient leading to the generation of cosmological magnetic fields. Finally, we will show that the existing upper limits on vorticity coming from CMB anisotropies impose a lower limit on the amplitude of the produced magnetic fields.

The power spectrum of super-Hubble fluctuations of $\nabla_{\nu} A^{\nu}$ produced during an inflationary phase characterized by a slow-roll parameter $\epsilon$ can be written as [9]

$$
P_{\nabla A}(k)=\frac{9 H_{k_{0}}^{4}}{16 \pi^{2}}\left(\frac{k}{k_{0}}\right)^{-4 \epsilon},
$$

where $H_{k_{0}}$ is the Hubble parameter when the $k_{0}$ mode left the horizon, and we have fixed $\xi=1 / 3$. As shown in [9], this value of $\xi$ corresponds to canonical normalization of commutation relations for creation and annihilation operators for states built out of the standard Bunch-Davies vacuum. Of course, in curved space-time it would be possible to choose a different normalization condition, but as long as it is a natural choice, we do not expect deviations from $\xi$ being of order unity and, thus, the results obtained in this work will remain essentially unchanged. The pivot point will be chosen as $k_{0} \simeq H_{0}$ with $H_{0}$ the Hubble parameter today. The corresponding field variance will read

$$
\left\langle\left(\nabla_{\mu} A^{\mu}\right)^{2}\right\rangle=\int_{k_{c}}^{H_{0}} \frac{d k}{k} \frac{9 H_{k_{0}}^{4}}{16 \pi^{2}}\left(\frac{k}{k_{0}}\right)^{-4 \epsilon} \simeq \frac{9 H_{k_{0}}^{4}}{64 \pi^{2} \epsilon}\left(\frac{H_{0}}{k_{c}}\right)^{4 \epsilon},
$$

where $k_{c}$ is the infrared cutoff which is usually set by the comoving Hubble radius at the beginning of inflation (see [15] and references therein for problems with infrared divergences during inflation). The above expression, for 
super-Hubble modes today, can be identified with the cosmological constant scale $M_{\Lambda} \simeq 2 \times 10^{-3} \mathrm{eV}$ and, thus,

$$
M_{\Lambda}^{4} \simeq \frac{9 H_{k_{0}}^{4}}{64 \pi^{2} \epsilon}\left(\frac{H_{0}}{k_{c}}\right)^{4 \epsilon} .
$$

Since $\epsilon$ is positive, we see that, in general, $H_{k 0} \lesssim M_{\Lambda}$. Notice that $\nabla_{\nu} A^{\nu}$ is constant on super-Hubble scales and starts decaying as $1 / a$ once the mode reenters the Hubble radius. Thus, today, a mode $k$ will have been suppressed by a factor $a_{\text {in }}(k)$ (we are assuming that the scale factor today is $\left.a_{0}=1\right)$. This factor will be given by $a_{\text {in }}(k)=\Omega_{M} H_{0}^{2} / k^{2}$ for modes entering the Hubble radius in the matter era, i.e. for $k<k_{\text {eq }}$ with $k_{\text {eq }} \simeq(14 \mathrm{Mpc})^{-1} \Omega_{M} h^{2}$ the value of the mode which entered at matter-radiation equality. For $k>k_{\text {eq }}$ we have $a_{\text {in }}(k)=\sqrt{2 \Omega_{M}}\left(1+z_{\text {eq }}\right)^{-1 / 2} H_{0} / k$. It is then possible to compute the corresponding power spectrum for the effective electric charge density today: $\rho_{g}^{0}=J_{\nabla \cdot A}^{0}=-\xi \partial_{0}\left(\nabla_{\nu} A^{\nu}\right)$. Thus from

$$
\left\langle\rho(\vec{k}) \rho^{*}(\vec{h})\right\rangle=(2 \pi)^{3} \delta(\vec{k}-\vec{h}) \rho^{2}(k)
$$

we define $P_{\rho}(k)=\frac{k^{3}}{2 \pi^{2}} \rho^{2}(k)$, which is given by

$$
P_{\rho}(k)= \begin{cases}0, & k<H_{0}, \\ \frac{\Omega_{M}^{2} H_{0}^{2} H_{k 0}^{4}}{16 \pi^{2}}\left(\frac{k}{k_{0}}\right)^{-4 \epsilon-2}, & H_{0}<k<k_{\mathrm{eq}}, \\ \frac{2 \Omega_{M} H_{0}^{2} H_{k 0}^{4}}{16 \pi^{2}\left(1+z_{\mathrm{eq}}\right)}\left(\frac{k}{k_{0}}\right)^{-4 \epsilon}, & k>k_{\mathrm{eq}} .\end{cases}
$$

Therefore the corresponding charge variance will read $\left\langle\rho^{2}\right\rangle=\int \frac{d k}{k} P_{\rho}(k)$. Notice that for modes entering the Hubble radius in the radiation era, the power spectrum is nearly scale invariant. Also, due to the constancy of $\nabla_{\nu} A^{\nu}$ on super-Hubble scales, the effective charge density power spectrum is negligible on such scales, so that we do not expect magnetic field nor vorticity generation on those scales. Notice that, on sub-Hubble scales, the present amplitude of the longitudinal electric fields would be precisely $E_{L} \simeq \nabla_{\nu} A^{\nu}$.

For an observer moving with the cosmic plasma with four-velocity $u^{\mu}$, it is possible to decompose the Faraday tensor in its electric and magnetic parts as $F_{\mu \nu}=$ $2 E_{[\mu} u_{\nu]}+\left(\epsilon_{\mu \nu \rho \sigma} / \sqrt{g}\right) B^{\rho} u^{\sigma}$, where $E^{\mu}=F^{\mu \nu} u_{\nu}$ and $B^{\mu}=\epsilon^{\mu \nu \rho \sigma} /(2 \sqrt{g}) F_{\rho \sigma} u_{\nu}$. In the infinite conductivity limit, Ohm's law $J^{\mu}-u^{\mu} u_{\nu} J^{\nu}=\sigma F^{\mu \nu} u_{\nu}$ implies $E^{\mu}=0$. Therefore, in that case the only contribution would come from the magnetic part. Here, $J^{\mu}$ is the current generated in the plasma which is assumed neutral, i.e. $J_{\mu} u^{\mu}=0$. Thus, from (4), we get

$$
F_{; \nu}^{\mu \nu} u_{\mu}=\frac{\epsilon^{\mu \nu \rho \sigma}}{\sqrt{g}} B_{\rho} u_{\sigma ; \nu} u_{\mu}=J_{\nabla \cdot A}^{\mu} u_{\mu}
$$

that for comoving observers in a Robertson-Walker metric imply (see also [14])

$$
\frac{1}{a^{2}} \vec{\omega} \cdot \vec{B}=\frac{\rho_{g}^{0}}{a^{2}},
$$

where $\vec{v}=d \vec{x} / d \eta, \vec{\omega}=\vec{\nabla} \times \vec{v}$ is the fluid vorticity, $\rho_{g}^{0}$ is the effective charge density today, and the $\vec{B}$ components scale as $B_{i} \propto 1 / a$ as can be easily obtained from $\epsilon^{\mu \nu \rho \sigma} F_{\rho \sigma ; \nu}=0$ to the lowest order in $v$. Thus, as commented before, the presence of the nonvanishing cosmic effective charge density necessarily creates both magnetic field and vorticity. In the absence of sources, vorticity scales as $a^{3 w-1}$ [16], with $w$ the equation of state parameter of the dominant component. However, due to the presence of the effective current, we find that vorticity grows as $|\vec{\omega}| \propto a$, from the radiation era until present.

Using (11), it is possible to estimate a lower limit on the present amplitude of the magnetic fields generated. Since we are not assuming any particular mechanism for the generation of the primordial magnetic and vorticity perturbations in the early Universe, we will consider them for simplicity as Gaussian stochastic variables such that

$$
\begin{aligned}
& \left\langle B_{i}(\vec{k}) B_{j}^{*}(\vec{h})\right\rangle=\frac{(2 \pi)^{3}}{2} P_{i j} \delta(\vec{k}-\vec{h}) B^{2}(k), \\
& \left\langle\omega_{i}(\vec{k}) \omega_{j}^{*}(\vec{h})\right\rangle=\frac{(2 \pi)^{3}}{2} P_{i j} \delta(\vec{k}-\vec{h}) \omega^{2}(k)
\end{aligned}
$$

with $B^{2}(k)=B k^{n}$ and $\omega^{2}(k)=\Omega k^{m}$ and where $P_{i j}=$ $\delta_{i j}-\hat{k}_{i} \hat{k}_{j}$ is introduced because of the transversality properties of $B_{i}$ and $\omega_{i}$. The spectral indices $n$ and $m$ are in principle arbitrary. Notice that when the plasma conductivity becomes large after reheating, we expect constraints on the power spectra coming from (11). We will be interested in calculating the mean fluctuation of the magnetic field in a region of size $\lambda$ using a Gaussian window function:

$$
B_{\lambda}^{2}=\frac{4 \pi}{(2 \pi)^{3}} \int \frac{d k}{k} k^{3} B k^{n} W^{2}(k \lambda),
$$

where $W(k \lambda)=\exp \left(-k^{2} \lambda^{2} / 2\right)$. Similar expressions can be written for $\omega_{\lambda}$ and $\rho_{\lambda}$. Thus from (11) it is possible to obtain $[14,17]$

$$
\rho_{\lambda}^{2}=\frac{1}{(2 \pi)^{3}} \frac{\Omega B}{2 \pi^{2}} S(\lambda, n, m)
$$

with

$$
\begin{aligned}
S(\lambda, n, m)= & \int_{H_{0}}^{\infty} \frac{d k}{k} k^{3} W^{2}(k \lambda) \\
& \times \int d^{3} p|\vec{k}-\vec{p}|^{m} p^{n}\left[1+(\vec{k}-\hat{\vec{p}} \cdot \hat{\vec{p}})^{2}\right],
\end{aligned}
$$

where $H_{0}<p<k_{c B}$ and $|\vec{k}-\vec{p}|>H_{0}$. For the upper cutoff of the magnetic power spectrum, we take a conservative value corresponding to the magnetic diffusion scale which is given by $k_{c B} \simeq 10^{11} \mathrm{Mpc}^{-1}$ [18] (a more detailed analysis can be found in [19]). Let us define

$$
G(\lambda, n)=\int_{k_{\min }}^{k_{\max }} \frac{d k}{k} k^{3} k^{n} W^{2}(k \lambda),
$$


where, as before, due to the vanishing of the charge density on super-Hubble scales, $k_{\min }$ is typically given by the comoving Hubble horizon at the time the fluctuations are evaluated and $k_{\max }=k_{c B}(\infty)$ in the magnetic (vorticity) cases, respectively. Thus, we finally obtain for the magnetic fluctuation on a scale $\lambda$

$$
B_{\lambda}^{2} \simeq \frac{4 \pi \rho_{\lambda}^{2} G(\lambda, n) G(\lambda, m)}{\omega_{\lambda}^{2} S(\lambda, n, m)} .
$$

Vorticity perturbations generate anisotropies in the CMB temperature at the recombination time whose amplitude should be compatible with present observations. Taking into account the scaling properties of vorticity derived before, such limits on a scale $\lambda$ can be written today as [14]

$$
\omega_{\lambda}^{2} \lesssim \frac{l^{2} C_{l} /(2 \pi) z_{\mathrm{rec}}^{2} G(\lambda, m)}{8 l^{3}(l+1) R(l, m)},
$$

where $l^{2} C_{l} /(2 \pi) \simeq 10^{-10}$ and

$$
R(l, m)=\int_{k_{\mathrm{rec}}}^{\infty} d k \frac{j_{l}^{2}\left(k \eta_{*}\right)}{\left(k \eta_{*}\right)^{2}} k^{m}
$$

with $\eta_{*}=\eta_{0}-\eta_{\text {rec }}$. We will consider the minimum of the right-hand side of (18) with respect to $l$ which, for $m<-1$, is located at $l \sim 29$ and, for $m>-1$, at $l \sim 1200$, which is the highest multipole measured by WMAP.

These stringent upper limits on vorticity can be translated using (17) into lower limits on the present value of the magnetic field created by the effective current. For the sake of concreteness, we take $H_{k 0} \simeq 2 \times 10^{-6} \mathrm{eV}$ in (9). This value corresponds to a scale of inflation around $100 \mathrm{GeV}$, i.e. in the electroweak range. It satisfies $H_{k 0} \lesssim M_{\Lambda}$ and also the limits on the primordial electromagnetic fluctuations coming from their imprint on CMB anisotropies (see [11]). We have evaluated numerically the integrals appearing in (17) for $\epsilon \simeq 0.01$, although the $\epsilon$ dependence of the bounds is very small. In Fig. 1 we show the lower limits on the magnetic fields generated by this mechanism on scales $\lambda=0.1 h^{-1} \mathrm{Mpc}$ [which is the relevant scale for galaxies and clusters (see [18])] and $\lambda=3000 h^{-1} \mathrm{Mpc}$. These results show that the produced fields could have strong amplitudes even in the largest scales and act as seeds for a galactic dynamo or even play the role of primordial fields and account for observations just by amplification due to the collapse and differential rotation of the protogalactic cloud.

It is interesting to note that, since super-Hubble modes of the effective electromagnetic current are not generated, we expect magnetic fields to be present only on subHubble scales. This means that the constraints coming from the dissipation of super-Hubble magnetic fields into
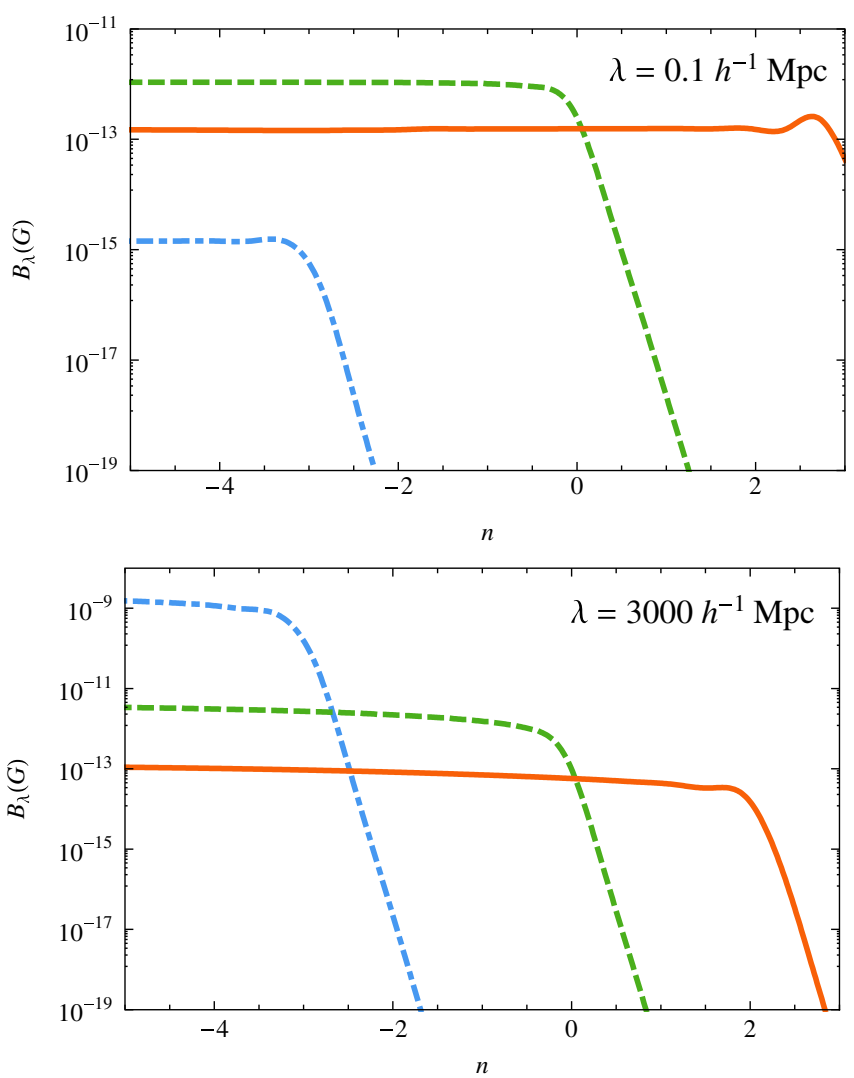

FIG. 1 (color online). Lower limits on the magnetic fields generated on galactic scales (upper panel) and Hubble horizon scales (lower panel) in terms of the magnetic spectral index $n$ for different values of the vorticity spectral index $m$. The dot-dashed blue curve is for $m=0$, the dashed green curve for $m \simeq-3$, and the full red curve for $m \simeq-5$.

gravity waves before nucleosynthesis [17] do not apply in the present case. In any case, these results show that a more precise determination of the magnetic and vorticity spectra on cosmological scales could help establish the feasibility of the extended theory in (1) for producing the observed cosmic magnetic fields.

We thank Ruth Durrer and Misao Sasaki for useful comments. This work has been supported by MICINN (Spain) Projects No. FIS 2008-01323 and No. FPA 200800592, CAM/UCM 910309, MEC Grant No. BES-200612059, and MICINN Consolider-Ingenio MULTIDARK CSD2009-00064. J.B. also received support from the Norwegian Research Council under the YGGDRASIL Project No. 195761/V11 and wishes to thank the hospitality of the University of Geneva where part of this work was performed. 
[1] L. M. Widrow, Rev. Mod. Phys. 74, 775 (2002); R. M. Kulsrud and E. G. Zweibel, Rep. Prog. Phys. 71, 046901 (2008); P. P. Kronberg, Rep. Prog. Phys. 57, 325 (1994).

[2] A. Neronov and I. Vovk, Science 328, 73 (2010); F. Tavecchio et al., arXiv:1004.1329; S. 'i. Ando and A. Kusenko, Astrophys. J. 722, L39 (2010); A. Neronov et al., arXiv:1006.0164.

[3] A. Brandenburg and K. Subramanian, Phys. Rep. 417, 1 (2005).

[4] M. L. Bernet, F. Miniati, S. J. Lilly, P. P. Kronberg, and M. Dessauges-Zavadsky, Nature (London) 454, 302 (2008); A. M. Wolfe, R. A. Jorgenson, T. Robishaw, C. Heiles, and J. X. Prochaska, Nature (London) 455, 638 (2008).

[5] E. R. Harrison, Mon. Not. R. Astron. Soc. 147, 279 (1970); Phys. Rev. Lett. 30, 188 (1973).

[6] M.S. Turner and L. M. Widrow, Phys. Rev. D 37, 2743 (1988); B. Ratra, Astrophys. J. 391, L1 (1992); K. Bamba and J. Yokoyama, Phys. Rev. D 69, 043507 (2004); K. Bamba and M. Sasaki, J. Cosmol. Astropart. Phys. 02 (2007) 030.

[7] J. M. Quashnock, A. Loeb, and D. N. Spergel, Astrophys. J. 344, L49 (1989); T. Vachaspati, Phys. Lett. B 265, 258 (1991).

[8] O. Bertolami and D. F. Mota, Phys. Lett. B 455, 96 (1999); A. L. Maroto, Phys. Rev. D 64, 083006 (2001); K. Ichiki et al., Science 311, 827 (2006).
[9] J. Beltrán Jiménez and A. L. Maroto, J. Cosmol. Astropart. Phys. 03 (2009) 016; Int. J. Mod. Phys. D 18, 2243 (2009).

[10] J. Beltrán Jiménez and A. L. Maroto, Phys. Lett. B 686, 175 (2010).

[11] J. B. Jimenez, T. S. Koivisto, A. L. Maroto, and D. F. Mota, J. Cosmol. Astropart. Phys. 10 (2009) 029.

[12] C. Itzykson and J.B. Zuber, Quantum Field Theory (McGraw-Hill, New York, 1980); N. N. Bogoliubov and D. V. Shirkov, Introduction to the Theory of Quantized Fields (InterScience, New York, 1959).

[13] A. Dolgov and J. Silk, Phys. Rev. D 47, 3144 (1993).

[14] C. Caprini and P. G. Ferreira, J. Cosmol. Astropart. Phys. 02 (2005) 006.

[15] Y. Urakawa and T. Tanaka, Prog. Theor. Phys. 122, 779 (2009).

[16] R. Durrer, The Cosmic Microwave Background (Cambridge University Press, Cambridge, England, 2008).

[17] C. Caprini and R. Durrer, Phys. Rev. D 65, 023517 (2001); C. Caprini, R. Durrer, and E. Fenu, J. Cosmol. Astropart. Phys. 11 (2009) 001; T. Kahniashvili et al., Phys. Rev. D 82, 083005 (2010); T. Kahniashvili, A. G. Tevzadze, and B. Ratra, Astrophys. J. 726, 78 (2011).

[18] R. Durrer, P. G. Ferreira, and T. Kahniashvili, Phys. Rev. D 61, 043001 (2000).

[19] K. Subramanian and J.D. Barrow, Phys. Rev. D 58, 083502 (1998). 